\title{
Diagnostic Accuracy of Magnetic Resonance Spectroscopy in Diagnosing Carcinoma Prostate
}

\author{
Fariha Mumtaz, ${ }^{1}$ Mudassar Saeed Pansota, ${ }^{1}$ Mumtaz Rasool ${ }^{1}$
}

\begin{abstract}
Background: Magnetic resonance spectroscopy (1H-MRS) produces a non-invasive analysis of the metabolism of the tissue, determining the relative concentrations of their metabolites and the interactions produced between them, which may be used in tumor diagnosis and showed good diagnostic accuracy for prostate cancer detection.

Objective: To determine the diagnostic accuracy of Magnetic Resonance Spectroscopy (MRS) in diagnosing prostate cancer.

Methodology: This was a cross sectional study, conducted at department of Radiology, Bahawal Vitoria Hospital, Bahawalpur, from July 2019 to June 2020. A total of 206 male patients with clinical suspicion of carcinoma prostate, 50-80 years of age will be included. Patients with already diagnosed carcinoma prostate, prostatic abscess, acute or chronic prostatitis were excluded. Each patient has undergone MRS examination. Each MRS was interpreted by one consultant radiologist and was looked for choline + creatine/citrate ratio for carcinoma prostate. Findings of MRS and histopathology were correlated. Data were analyzed by using SPSS 20.

Results: In 120 MRS-positive patients, 108 were True Positive and 12 were False Positive. Among, 86 MRS negative patients, 16 were False Negative whereas 70 were True Negative. Overall sensitivity, specificity, positive predictive value, negative predictive value, and diagnostic accuracy of magnetic resonance spectroscopy (MRS) in diagnosing carcinoma prostate was $87.10 \%, 85.37 \%, 90.0 \% 81.40 \%$, and $86.41 \%$ respectively.

Conclusion: This study concluded that Magnetic Resonance Spectroscopy is the non-invasive modality of choice with high diagnostic accuracy in detecting prostate cancer.
\end{abstract}

Keywords: Prostate cancer, Magnetic Resonance Spectroscopy, Sensitivity.

Article Citation: Mumtaz F, Pansota MS, Rasool M. Diagnostic Accuracy of Magnetic Resonance Spectroscopy in Diagnosing Carcinoma Prostate, JSZMC 2021;12(3):12-16. DOI: https://doi.org/10.47883/jszmc.v12i3.111

This Open Access Article in Journal of Sheikh Zayed Medical College is licensed under a Creative Commons Attribution- 4.0 International License (CC BY 4.0).

\section{Introduction}

Prostate cancer is the second most common cause of cancer deaths in men, with an estimated 41,000 deaths and more than 125,000 new cases per year. ${ }^{1}$ Currently it is the most common male malignancy and the majority of cases are diagnosed at a time when tumor has extended beyond the confines of the gland, making it incurable. ${ }^{2}$ The evidence has shown the prevalence of prostate carcinoma as $49 \%{ }^{3}$ The specific causes of prostate cancer remain unknown. The primary risk factors are age and family history. Prostate cancer is very uncommon in men younger than 45 but becomes more common with advancing age. The average age at the time of diagnosis is 70 years. ${ }^{4}$

Different Protocols and screening tests are being used worldwide for its early detection. The most commonly accepted protocol being practiced is a clinical diagnosis based on Digital Rectal Examination, screening by serum Prostate Specific
Antigen (PSA), and Transrectal Ultrasonography (TRUS). ${ }^{4,5}$ The false-negative rate of TRUS-guided biopsies is estimated to be between $15 \%$ to $34 \%{ }^{6}$ Magnetic Resonance (MR) Imaging as a noninvasive tool plays an increasingly important role in the detection, localization, and staging of prostate cancer. It has the potential to improve the sensitivity and specificity for detecting PCa and promises to make it a successful imaging tool for improving many aspects of PCa management. ${ }^{7,8}$

In vivo proton MR spectroscopy (1H-MRS) produces a noninvasive analysis of the metabolism of the tissue, determining the relative concentrations of their metabolites and the interactions produced between them, which may be used in tumor diagnosis and has been proved to be a sensitive method in identifying carcinoma prostate. ${ }^{9,10}$ The levels of citrate, choline, and creatine are useful for the evaluation of prostate cancer, as it is known that tumors have elevated

1. Department of Radiology, Bahawal Victoria Hospital/Quaid-e-Azam Medical College, Bahawalpur, Pakistan.

2. Department of Urology,Shahida Islam Medical College, Lodhran. Pakistan.

3. Department of Urology, Bahawal Victoria Hospital/Quaid-e-Azam Medical College, Bahawalpur, Pakistan.

Correspondence: Dr. Mudassar Saeed Pansota, Assistant Professor, Department of Urology, Shahida Islam Medical College, Lodhran. Pakistan. Email: netygeulis749@hotmail.com Received: 27-06-2020 Published: 24-09-2021 
levels of choline and a decreased levels of citrate. Though separate analysis of creatine and choline could not be possible, so choline + creatine/citrate ratio can be used for the prediction of prostatic malignancy. ${ }^{6,9}$ The aim of this study was to determine the diagnostic accuracy of Magnetic Resonance Spectroscopy (MRS) in diagnosing prostate cancer. If its diagnostic accuracy is found high, then our general population may be provided with a non-invasive screening test for prostate cancer when it is localized and thus at a curable stage, so clinicians could take early management measures to limit the disease progression in order to reduce the morbidity and mortality of these particular patients.

\section{Methodology}

This descriptive, cross-sectional study was done on 206 male patients with clinical suspicion of carcinoma prostate (having enlarged prostate with hard consistency, irregular surface, rectal mucosa not mobile and nodule on Digital Rectal Examination and S/PSA $>4 \mathrm{ng} / \mathrm{ml}$ ) and age between 50-80 years who were presented at the department of Radiology, Bahawal Vitoria Hospital, Bahawalpur, were selected for the study. Duration of study was from July 2019 to June 2020. Patients having acute or chronic prostatitis, prostatic abscess, and contraindication to MRS i.e. MRS incompatible prosthesis or cardiac pacemaker holders were excluded from the study. Informed written consent from each patient was taken and ethical approval was sought from Institutional Ethical Committee. After this, proton Magnetic Resonance Spectroscopy (1H MRS) was performed in every patient using 1.5 Tesla MR system with a gradient strength of $33 \mathrm{mT} / \mathrm{m}$. A fast scout scan in sagittal, axial, and coronal planes was obtained. The scanning technique used was the point-resolved spectroscopy single-voxel technique. It was followed by water suppression pulses to be followed by data acquisition. Each MRS was interpreted by one consultant radiologist (with post-fellowship experience of at least 10 years) and was looked for creatine/citrate ratio and taken as positive if there was $>1.2$ Magnetic Resonance Spectroscopy. Magnetic Resonance Spectroscopy findings were correlated with the histopathology report.

Collected data was analyzed through computer software SPSS 20.0. Mean and standard deviation was calculated for quantitative variables. Frequency and percentage were calculated for qualitative variables, and a $2 \times 2$ contingency table was used to calculate sensitivity, specificity, positive predictive value, negative predictive value, and diagnostic accuracy of MRS in diagnosing carcinoma prostate, taking histopathology as a gold standard.

\section{Results}

The age range in this study was from $50-80$ years with a mean age of $66.57 \pm 7.44$ years. The majority of the patients $117(56.80 \%)$ were between 66 to 80 years of age. Out of these 206 patients, 133 (64.56\%) were between 4-12 months of duration of disease with mean duration of disease was $11.63 \pm 4.44$ months. The mean S/PSA was $22.14 \pm 10.88 \mathrm{ng} / \mathrm{ml}$. All the patients were subjected to Magnetic Resonance Spectroscopy. MRS supported the diagnosis of prostate cancer in $120(58.25 \%)$ patients. Histopathology confirmed prostate cancer in $124(60.19 \%)$ cases whereas $82(39.81 \%)$ patients revealed no prostate cancer. In 120 MRS-positive patients, 108 (True Positive) had prostate cancer and 12 (False Positive) had no prostate cancer on histopathology. Among, 86 MRS negative patients, 16 (False Negative) had prostate cancer on histopathology whereas 70 (True Negative) had no prostate cancer on histopathology. Overall sensitivity, specificity, positive predictive value, negative predictive value, and diagnostic accuracy of Magnetic Resonance Spectroscopy (MRS) in diagnosing carcinoma prostate, taking histopathology as the gold standard was $87.10 \%$, $85.37 \%, 90.0 \% 81.40 \%$, and $86.41 \%$ respectively.

Figure-I: Diagnostic accuracy of Magnetic Resonance Spectroscopy (MRS) in diagnosing Carcinoma Prostate, taking Histopathology as Gold Standard.

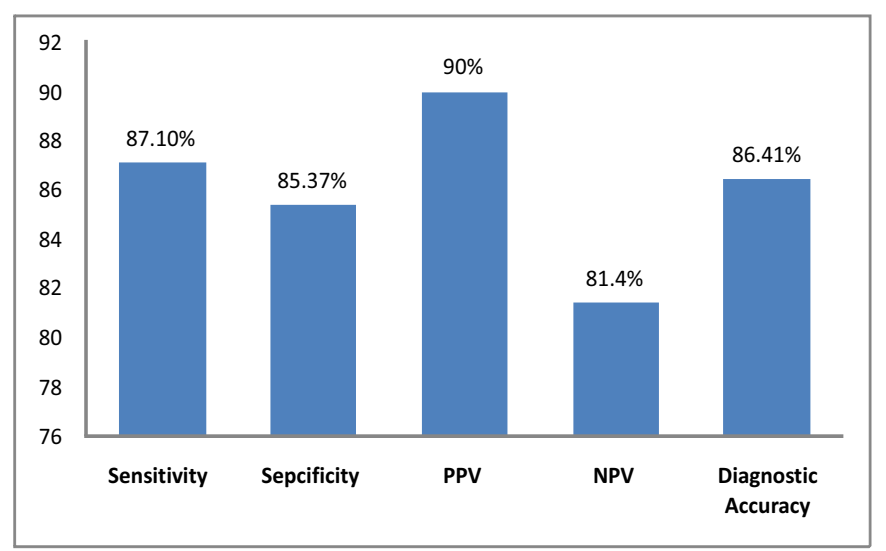




\section{Discussion}

Molecular imaging is emerging as an important and promising tool for the development of anticancer therapies. Like Positron Emission Tomography (PET), Magnetic Resonance Spectroscopy (MRS) is a non-invasive technique that produces an image of function rather than of anatomy, thus apparently providing better accuracy in monitoring early response to antiproliferative treatment in several clinical settings. Moreover, its ability to distinguish metabolite levels is proving useful in the management of specific cancers. MRS imaging also has the potential to significantly improve the metabolic characterization of prostate cancer in patients before and after therapy. A study presented the results showing the potential clinical application of $1 \mathrm{H}-\mathrm{MRS}$ imaging in the evaluation of the prostate with an endorectal detection coil at a high magnetic field strength of $3 \mathrm{~T}^{8,9}$ This study was conducted to determine the diagnostic accuracy of magnetic resonance spectroscopy (MRS) in diagnosing carcinoma prostate, taking histopathology as a gold standard.

In this study, sensitivity, specificity, positive predictive value, negative predictive value, and diagnostic accuracy of Magnetic Resonance Spectroscopy (MRS) in diagnosing carcinoma prostate, taking histopathology as gold standard was $87.10 \%, 85.37 \%, 90 \%, 81.40 \%$, and $86.41 \%$ respectively. The sensitivity and specificity of MR Spectroscopy (choline + creatine/citrate ratio $>$ 1.5) for diagnosing prostate cancer as observed by Caivano $\mathrm{R}$ et $\mathrm{al}^{9}$ was $92 \%$ and $89 \%$ respectively while Testa $\mathrm{C}$ et $\mathrm{al}^{10}$ has shown this sensitivity and specificity (choline + creatine/citrate ratio $>1.2$ ) as $70 \%$ and $44 \%$ respectively.

Magnetic Resonance Spectroscopy of the prostate increases the diagnostic probability in cases of cancer, by adding metabolic data on the gland to the morphological information. The sensitivity of this method ranges from $68 \%$ to $95 \%$ and specificity, from $70 \%$ to $91 \%$. $^{11,12}$ Advantages of the utilization of this technique in the determination of prostate cancer include accurate spectral localization of each small morphologically abnormal region; precise correlation between the spectral mapping and the high-resolution Magnetic Resonance Imaging; evaluation of the abnormal metabolism extent; three-dimensional coverage of the entire gland. ${ }^{13}$

A variation is observed when MRI results and MRSI metabolic data are combined. Together, they result in $56-94 \%$ sensitivity and $70-98 \%$ specificity. ${ }^{12,14}$ In a study, Yuen et $\mathrm{al}^{15}$ observed that MRI data in association with those of MRSI, presented $100 \%$ sensitivity and $70.3 \%$ specificity in the determination of suspicious areas. Recently Prando et $\mathrm{al}^{16}$ observed that MRI combined with MRSI presented high sensitivity (84\% to $100 \%$ ) and low specificity $(44 \%$ to $71 \%$ ) in the identification of target areas. The combination of MRS and T2WI detected prostate tumors with a specificity of 79-93\% and sensitivity of $72-89 \%{ }^{17,18}$ compared to radical prostatectomy, although no incremental benefit of adding MRS to T2WI was seen in a prospective trial of 110 men (ROC-AUC 0.60 vs 0.58 respectively). ${ }^{19}$ In detecting tumors of $>3 \mathrm{~mm}$ diameter in the peripheral zone, MRS had a high specificity of $98 \%$ (compared to $83 \%$ for T2WI and $94 \%$ DCEI) but at the cost of poor sensitivity of $53 \%$ when used alone (compared to $94 \%$ for T2WI and $56 \%$ for DCEI). ${ }^{19}$

A meta-analysis ${ }^{20}$ identified 31 test-accuracy studies (1765 patients); 16 studies (17 populations) with a total of 581 patients were suitable for meta-analysis. Nine combined MRI/MRSI studies (10 populations) examining men with pathologically confirmed prostate cancer (297 patients; 1518 specimens) had a pooled sensitivity and specificity on prostate subpart level of $68 \%(95 \% \mathrm{CI}, 56-78 \%)$ and $85 \%$ (95\% CI, $78-90 \%$ ), respectively. Compared with patients at high risk for clinically relevant cancer (six studies), sensitivity was lower in low-risk patients (four studies) (58\% [46-69\%] vs 74\% [58-85\%]; $p>0.05)$ but higher for specificity (91\% [86-94\%] vs 78\% [70-84\%]; $\mathrm{p}<0.01)$. Seven studies examining patients with suspected prostate cancer at combined MRI/MRSI (284 patients) had an overall pooled sensitivity and specificity on patients level of $82 \%$ $(59-94 \%)$ and $88 \%(80-95 \%)$. In the low-risk group (five studies) these values were $75 \%(39-93 \%)$ and $91 \%(77-97 \%)$, respectively. ${ }^{17,20}$

Lawrentschuk $\mathrm{N}$ et $\mathrm{al}^{21}$ undertook a review of studies of MRI or MRS which recruited participants with a previous negative biopsy and persistently elevated PSA. For MRI or combined MRI and MRS, they reported a sensitivity of $57 \%$ to $100 \%$ and a specificity of $44 \%$ to $96 \%$. The authors found that $54 \%$ of patients $(34 / 63)$ were diagnosed with cancer 
solely based on a MRI-targeted biopsy. In a study done by Hasumi et $\mathrm{al}^{22}$ thirteen out of 19 voxels showed a cancer pattern which indicated a high choline peak and low citrate peak the accuracy, sensitivity, and specificity of MRS diagnosis of tumor localization were $84.2 \%, 81.3 \%$, and $100 \%$, respectively.

\section{Conclusion}

This study concluded that Magnetic Resonance Spectroscopy is the non-invasive modality of choice with high diagnostic accuracy in detecting prostate cancer. So, we recommend that Magnetic Resonance Spectroscopy should be used routinely in all suspected cases of prostate cancer for accurate assessment of prostate cancer when it is localized and thus at a curable stage in order to reduce the morbidity and mortality of these particular patients.

Authors Contribution: FM: Conception of work and Revising. MSP: Design of work, Acquisition and analysis of data and Drafting. MR: Design of work and Drafting.

All authors critically revised and approve its final version.

Conflict of Interest: Author has declared no conflict of interest.

Sources of Funding: The source of funding was self.

\section{Disclaimer: None}

\section{References}

1. Rasool M, Pansota MS, Tabassum SA, Saleem MS. Frequency of carcinoma of prostate in clinically benign prostatic hyperplasia and role of different screening tests. Pak Armed Forces Med J.2012; 62(3):431-34.

2. Roethke M, Anastasiadis AG, Lichy M, Werner M, Wagner P, Kruck S. MRI-guided prostate biopsy detects clinically significant cancer: analysis of a cohort of 100 patients after previous negative TRUS biopsy. World J Urol. 2012; 30:213-18.

3. Sciarra A, Panebianco V, Ciccariello M, Salciccia S, Cattarino S, Lisi D, et al. Value of magnetic resonance spectroscopy imaging and dynamic contrast-enhanced imaging for detecting prostate cancer foci in men with prior negative biopsy. Clin Cancer Res. 2010; 16:1875-83.
4. Panebianco V, Sciarra A, Ciccariello M, Lisi D, Bernardo S, Cattarino S, et al. Role of magnetic resonance spectroscopic imaging $([1 \mathrm{H}] \mathrm{MRSI})$ and dynamic contrast-enhanced MRI (DCE-MRI) in identifying prostate cancer foci in patients with negative biopsy and high levels of prostate specific antigen (PSA). Radiol Med. 2010; 115:1314-29.

5. Hoeks CMA, Schouten MG, Bomers JGR, Hoogendoorn SP, Hulsbergen-van de Kaa CA, Hambrock T, et al. Three-Tesla magnetic resonanceguided prostate biopsy in men with increased prostate specific antigen and repeated, negative, random, systematic, transrectal ultrasound biopsies: detection of clinically significant prostate cancers. Eur Urol. 2012; 62:902-9.

6. Tempany C, Franco F. Prostate MRI: update and current role. Applied Radiol. 2012; 41(3):17-22.

7. Hambrock T, Somford DM, Hoeks C, Bouwense SA, Huisman H, Yakar D, et al. Magnetic resonance imaging guided prostate biopsy in men with repeat negative biopsies and increased prostate specific antigen. JUrol. 2010; 183:520-7.

8. Villeirs GM, De Meerleer GO, De Visschere PJ, Fonteyne VH, Verbaeys AC, Oosterlinck W. Combined magnetic resonance imaging and spectroscopy in the assessment of high-grade prostate carcinoma in patients with elevated PSA: a single-institution experience of 356 patients. Eur J Radiol.2011; 77:340-5.

9. Caivano R, Cirillo P, Balestra A, Lotumolo A, Fortunato G, Macarini L, et al. Prostate cancer in magnetic resonance imaging: diagnostic utilities of spectroscopic sequences. J Med Imaging Radiat Oncol. 2012; 56(6):606-16.

10.Testa C, Schiavina R, Lodi R, Salizzoni E, Tonon C, D'Errico A, et al. Accuracy of MRI/MRSI-based transrectal ultrasound biopsy in peripheral and transition zones of the prostate gland in patients with prior negative biopsy. NMR Biomed. 2010;23:1017-26.

11. Shukla-Dave A, Hricak H, Eberhardt SC. Chronic prostatitis: MR imaging and $1 \mathrm{H}$ MR spectroscopic imaging findings - initial observations. Radiology. 2004; 231:717-24.

12.Kurhanewicz J, Vigneron DB, Hricak H. Threedimensional H-1 MR spectroscopic imaging of the in situ human prostate with high $(0.24-0.7 \mathrm{~cm} 3)$ spatial resolution. Radiology. 1996; 198:795-805.

13. Ackerstaff E, Pflug BR, Nelson JB. Detection of increased choline compounds with proton nuclear magnetic resonance spectroscopy subsequent to malignant transformation of human prostatic epithelial cells. Cancer Res. 2001; 61:3599-603. 
14. Zakian KL, Eberhardt S, Hricak H. Transition zone prostate cancer: metabolic characteristics at $1 \mathrm{H}$ MR spectroscopic imaging - initial results. Radiology. 2003; 229:241-7.

15. Yuen JS, Thng CH, Tan PH. Endorectal magnetic resonance imaging and spectroscopy for the detection of tumor foci in men with prior negative transrectal ultrasound prostate biopsy. J Urol. 2004;171:1482-6.

16. Prando A, Kurhanewicz J, Borges AP. Prostatic biopsy directed with endorectal MR spectroscopic imaging findings in patients with elevated prostate specific antigen levels and prior negative biopsy findings: early experience. Radiology. 2005; 236:903-10.

17.Villeirs GM, De Meerleer GO, De Visschere PJ, Fonteyne VH, Verbaeys AC, Oosterlinck W. Combined magnetic resonance imaging and spectroscopy in the assessment of high grade prostate carcinoma in patients with elevated PSA: a single-institution experience of 356 patients. Eur J Radiol 2011; 77: 340-345.

18. Tanimoto A, Nakashima J, Kohno H, Shinmoto H, Kuribayashi S. Prostate cancer screening: the clinical value of diffusion-weighted imaging and dynamic MR imaging in combination with T2weighted imaging. J MagnReson Imaging 2007; 25: 146-152.
19. Weinreb JC, Blume JD, Coakley FV. Prostate cancer: sextant localization at MR imaging and MR s pectros copic imaging before prostatectomy-results of ACRIN prospective multi-institutional clinico-pathologic study. Radiology 2009; 251:122-133.

20. Umbehr M, Bachmann LM, Held U, Kessler TM, Sulser T, Weishaupt D, et al. Combined magnetic resonance imaging and magnetic resonance spectroscopy imaging in the diagnosis of prostate cancer: a systematic review and meta-analysis. Eur Urol. 2009; 55:575-591.

21. Lawrentschuk N, Fleshner N. The role of magnetic resonance imaging in targeting prostate cancer in patients with previous negative biopsies and elevated prostate-specific antigen levels. BJU Int. 2009; 103:730-3.

22. Hasumi M, Suzuki K, Oya N, Ito K, Kurokawa K, Fukabori Y, Yamanaka H. MR spectroscopy as a reliable diagnostic tool for localization of prostate cancer. Anticancer Res. 2002 Mar-Apr; 22(2B):1205-8. 